\title{
New Evaluation Technique for WTG Design Wind Speed Using a CFD-Model-Based Unsteady Flow Simulation with Wind Direction Changes
}

\author{
Takanori Uchida, ${ }^{1}$ Takashi Maruyama, ${ }^{2}$ and Yuji Ohya ${ }^{1}$ \\ ${ }^{1}$ Research Institute for Applied Mechanics (RIAM), Kyushu University, 6-1 Kasuga-kouen, Kasuga, Fukuoka 816-8580, Japan \\ ${ }^{2}$ Disaster Prevention Research Institute (DPRI), Kyoto University, Gokashyo, Uji, Kyoto 611-0011, Japan \\ Correspondence should be addressed to Takanori Uchida, takanori@riam.kyushu-u.ac.jp
}

Received 11 November 2010; Accepted 22 February 2011

Academic Editor: Guan Yeoh

Copyright (C) 2011 Takanori Uchida et al. This is an open access article distributed under the Creative Commons Attribution License, which permits unrestricted use, distribution, and reproduction in any medium, provided the original work is properly cited.

\begin{abstract}
Because a significant portion of the topography in Japan is characterized by steep, complex terrain, which results in a complex spatial distribution of wind speed, great care is necessary for selecting a site for the construction of wind turbine generators (WTG). We have developed a CFD model for unsteady flow called RIAM-COMPACT (Research Institute for Applied Mechanics, Kyushu University, computational prediction of airflow over complex terrain). The RIAM-COMPACT CFD model is based on large eddy simulation (LES). The computational domain of RIAM-COMPACT can extend from several meters to several kilometers, and RIAM-COMPACT can predict airflow and gas diffusion over complex terrain with high accuracy. The present paper proposes a technique for evaluating the deployment location of a WTG. The proposed technique employs the RIAM-COMPACT CFD model and simulates a continuous wind direction change over 360 degrees.
\end{abstract}

\section{Introduction}

With the implementation of the revised Building Standard Law of Japan in June 2007, all structures which exceed a height of $60 \mathrm{~m}$ are now subject to a performance assessment by a designated institution and the approval of the Minister of Land, Infrastructure, Transport, and Tourism. These requirements are in addition to the application for the approval of the structure as stipulated by the prerevised law. In the case of wind turbine generators, these revisions to the law call for calculations of the reference wind speed at the wind turbine hubheight, which is relevant for the wind resistant design of turbines. Accordingly, the ratio of the wind speed at the hubheight subject to topographical effects to the wind speed at the hubheight free of topographical effects (fractional increase of hubheight wind speed), hereafter, needs to be evaluated by taking topographical factors into consideration. Given this background, the "Guidelines for the Design of Wind Turbine Support Structures/Commentary" [1] were published by the Japan
Society of Civil Engineers in 2007. The published Guidelines are based on the "Building Standard Law of Japan" [2] and the "Architectural Institute of Japan Recommendations for Loads on Buildings/Commentary" [3]. The Guidelines recommend the use of a numerical model for each of the 16 wind directions to evaluate the design wind speed for wind turbines.

In the meantime, we have proposed a technique to calculate the reference wind speed for use in the wind-resistant design of turbines [4]. This technique ensures operational safety of the wind turbines and is able to take into account high-wind-speed conditions which are similar to those observed in reality. Specifically, typhoons which have hit the site of interest in the past with high wind speed are simulated with a mesoscale meteorological model, and the directions from which high wind speeds are anticipated are identified. Subsequently, an analysis is performed for the identified wind directions using the RIAM-COMPACT computational fluid dynamics (CFD) model [5]. For that analysis, the influence of a continuous wind direction change 
on the occurrence of high wind speed is evaluated from the analysis with the mesoscale meteorological model. However, in mesoscale meteorological models, numerous meteorological phenomena such as cumulus convection and rainfall are frequently modeled with a spatial resolution of a few hundred meters to a few kilometers (known as "parameterization" in the field of meteorology). Therefore, the spatial resolutions of mesoscale meteorological models are usually not high enough to be able to take into account the influence of the surrounding topography on the wind turbines, which is required for the wind-resistant design of the wind turbines. Furthermore, when coarse computational grids are used in a mesoscale meteorological model, wind velocity fluctuations cannot be simulated sufficiently. Even when high-resolution computational grids are used, as long as wind-direction-dependent simulations are performed, fine-scale variations of wind direction and speed due to the influence of the surrounding topography cannot easily be simulated with an accuracy larger than the angular resolution of the input wind directions (e.g., $22.5^{\circ}$ for a 16 wind direction simulation).

In this paper, a technique is proposed which resolves the above-mentioned drawbacks of the use of a mesoscale meteorological model. This technique utilizes the strengths of a CFD model, which is able to simulate unsteady turbulence fields, and the wind speed for the wind-resistant design of wind turbines is evaluated for all wind directions using a calculation in which the wind direction is varied continuously in time.

\section{Numerical Computation Method}

2.1. CFD Model. In this study, the RIAM-COMPACT natural terrain version model [5] is used in order to avoid numerical instability and to predict the airflow over complex terrain with high accuracy. The RIAM-COMPACT natural terrain version model uses collocated grids in a general curvilinear coordinate system. The velocity components and pressure are defined at the cell centers, and variables which result from the covariant velocity components multiplied by the Jacobian are defined at the cell faces. As for the computational technique, the finite-difference method (FDM) is adopted, and an LES model is used for the turbulence model. In LES, a spatial filter is applied on the flow field to separate eddies of various scales into grid-scale (GS) components, which are larger than the computational grids, and subgrid-scale (SGS) components, which are smaller than the computational grids. Largescale eddies, that is, the GS components of turbulence eddies, are numerically simulated directly without relying on the use of a physically simplified model. The main effect of small-scale eddies, that is, the SGS components, is to dissipate energy, and this dissipation is modeled based on the physical considerations of the SGS stress. For the governing equations of the flow, a spatially filtered continuity equation for incompressible fluid and a spatially-filtered Navier-Stokes equation are used. Because the present study is on airflow prediction in high-wind conditions, the effect of the temperature stratification which is generally present in the atmosphere is neglected.

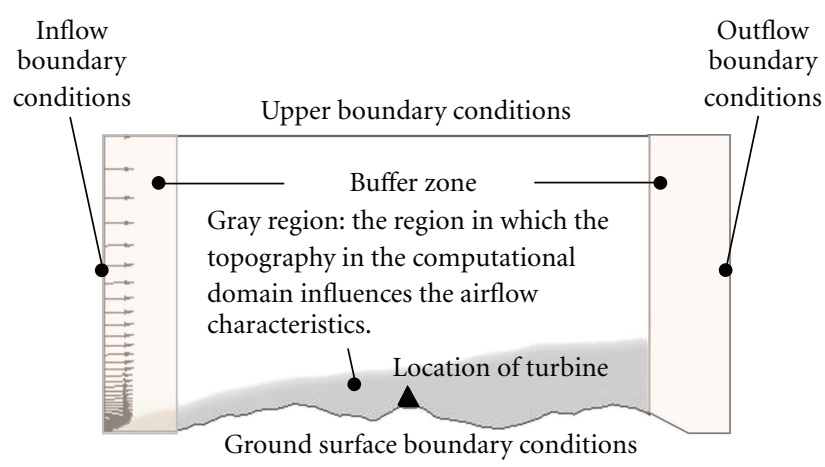

Terrain around the turbine is represented in detail.

FIgure 1: Computational domain and boundary conditions.

The computational algorithm and the time-marching method are based on a fractional step (FS) method and the Euler explicit method, respectively. The Poisson's equation for pressure is solved by the successive overrelaxation (SOR) method. For discretization of all the spatial terms except for the convective term, a second-order central difference scheme is applied. For the convective term, a third-order upwind difference scheme is applied. An interpolation technique based on 4-point differencing and 4-point interpolation by Kajishima [6] is used for the fourth-order central differencing that appears in the discretized form of the convective term. In the weighting of the numerical dispersion term of the third-order upwind differencing, $\alpha=3.0$ is commonly applied in the Kawamura-Kuwahara Scheme [7]. However, $\alpha$ is set to 0.5 in the present study to minimize the influence of numerical dispersion. For LES subgrid-scale modeling, the commonly used Smagorinsky model [8] is adopted. A wall-damping function is used with a model coefficient of 0.1. We have developed an LES-based model for analyzing neutral flow over variable orography and applied it to the problem of proper site selection. Model performance was evaluated using data from wind tunnel tests over simple geometries and from a real site [5].

2.2. Computational Conditions. The topography in the computational domain is reconstructed using mainly the $50-\mathrm{m}$ elevation data of the Geography Survey Institute of Japan. Fine grid spacing is adopted near the wind turbines in order to reconstruct the topographical features in detail. The computational domain is set up in such a way that airflow characteristics at the turbine location are subject to topographical influences (upwind zone) and that eddies flow out of the computational domain smoothly, and airflow at the turbine location is free from the influence of the outflow boundary (leeward zone) (Figure 1). For analyses of airflow over steep topography, a buffer zone is established which surrounds the computational domain. The terrain in the buffer zone is flat with an elevation close to zero meters and connects smoothly to the terrain in the computational domain.

As for the boundary conditions, slip conditions are applied to both the upper boundary and the side boundaries. For the upper boundary, the vertical gradient of the 


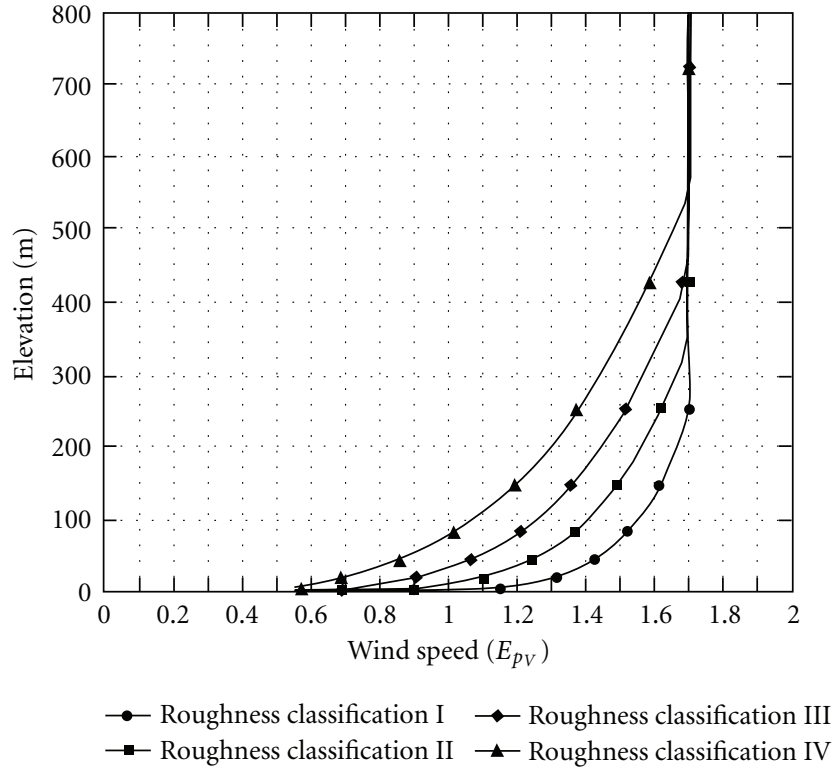

Figure 2: Vertical profiles of horizontal wind speed calculated from equations given in Bulletin no. 1454 of the Ministry of Construction, 2000.

TABLE 1: Coefficients for the vertical profiles of the mean horizontal wind speed which appear in Figure 2 (height correction coefficients for the mean horizontal wind speed $E_{p v}$.)

\begin{tabular}{lccc}
\hline Roughness classification & $Z_{b}(\mathrm{~m})$ & $Z_{G}(\mathrm{~m})$ & $\alpha$ \\
\hline I & 5 & 250 & 0.10 \\
II & 5 & 350 & 0.15 \\
III & 5 & 450 & 0.20 \\
IV & 10 & 550 & 0.27 \\
\hline
\end{tabular}

For height $\mathrm{H}$ smaller than $Z_{b}: E_{p v}=1.7\left(Z_{\mathrm{b}} / Z_{\mathrm{G}}\right)^{\alpha}$

For height $\mathrm{H}$ larger than $Z_{b}: E_{p v}=1.7\left(H / Z_{G}\right)^{\alpha}$

The values of $Z_{b}$ and $Z_{G}$ in the table are used in the formula.

horizontal wind speed components $(\bar{u}, \bar{v})$ and the vertical wind velocity component $(\bar{w})$ are all set to zero. For the side boundaries, the lateral gradient of the streamwise wind velocity component $(\bar{u})$, that of the vertical wind velocity component $(\bar{w})$, and that of the spanwise wind velocity component $(\bar{v})$ are all set to zero. For the inflow boundary condition, a vertical profile of the mean horizontal wind speed which is based on Bulletin no. 1454 of the Ministry of Construction, is applied according to the roughness classification (Figure 2 and Table 1). Furthermore, a convective outflow condition and the no-slip condition are imposed for the outflow boundary and ground surface, respectively. Changes in the wind direction are taken into account in the calculations of the technique proposed in the present study. Therefore, inflow boundary conditions are used when the flow is directed into the computational domain through the side boundary, and outflow boundary conditions are used when the flow is directed out of the computational domain through the side boundary.
TABLE 2: Details of computational domain.

(i) Computational domain: $8,500 \mathrm{~m}$ square wind farm with the observation pole located at the center. The vertical height of the computational domain is approximately $2,900 \mathrm{~m}$

(ii) Maximum elevation: $537 \mathrm{~m}$

(iii) Minimum elevation: $0 \mathrm{~m}$

(iv) Number of computational grids: $101 \times 101 \times 51$

(v) Number of computational grids including those in the buffer zone: $121 \times 121 \times 51$

(vi) Grid widths: $32 \mathrm{~m} \sim 769 \mathrm{~m}$ (horizontal direction); $1.7 \mathrm{~m}$

$\sim 347 \mathrm{~m}$ (vertical direction)

(vii) Reynolds number: $\operatorname{Re}=10^{4}$ (based on the difference between the minimum and maximum elevations and the wind speed at the maximum elevation.)

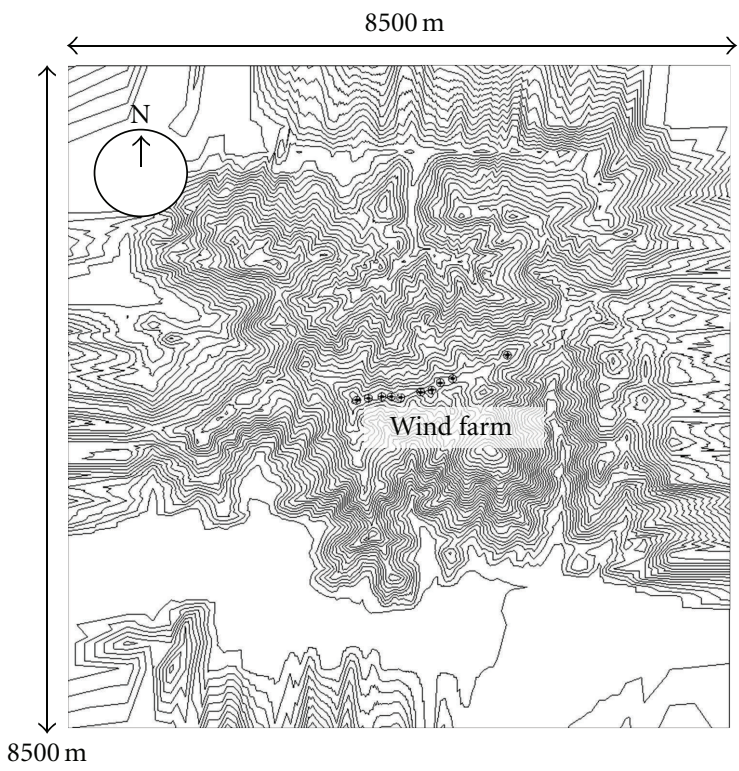

Figure 3: Wind turbine locations and computational domain.

\section{An Example of Design Wind Speed Evaluation}

3.1. Summary of Wind Farm. In this section, the proposed technique is applied to evaluate the appropriate wind speed for wind-resistant design of turbines for a wind farm in Japan. The evaluation is made for a wind farm which consists of ten wind turbines with rotor diameters of $62 \mathrm{~m}$ (Figure 3 ). The hubheight and the power output of each of the turbines are $60 \mathrm{~m}$ and $1.3 \mathrm{MW}$, respectively. Figure 3 shows the details of the computational domain (see Table 2).

3.2. Wind Direction Change. For the inflow profile, a vertical profile of the horizontal mean wind speed for roughness classification III is selected according to the previous literature [1]. This selection is based on the surface roughness around the wind turbine. Using the same vertical profile of wind speed, a simulation is performed in which the wind profile is rotated continuously over 360 degrees (Figure 4). In order to ensure that the wind direction change has little influence 
on the statistical values calculated over approximately 10minute intervals, the number of time steps required for the rotation of the wind profile over 360 degrees is set to an appropriate value. This value is selected according to the results of an investigation on the influence of the rate of wind direction change on the simulation results. In this investigation, which is conducted prior to the main simulation, the results of a simulation with a fixed vertical profile of wind speed rotated over 360 degrees are compared to the results of simulations with two different settings: (1) the number of time steps for the wind profile rotation is increased to 10 times that of the original; (2) the wind profile does not rotate, that is, wind flow comes from a fixed direction. In this study, 400,000 time steps are used to rotate the wind direction over $360 \mathrm{deg}\left(9 \times 10^{-4}\right.$ degs/step $)$ with a dimensionless time interval of 0.002 (approximately 0.1 seconds in actual time). For the simulation, parallel computation was performed using a single computer with an Intel Core 2 Extreme 6850 ( $3 \mathrm{GHz}, 4$ core), $2 \mathrm{~GB}$ of memory, Intel Fortran v.10.1.025, and Windows XP SP3. The entire simulation required 70,790s (approximately 20 hours) for the wind direction change of 360 degrees (400,000 time steps).

In the simulation, some fluctuations are observed immediately after the initiation of the simulation. This phenomenon implies that it takes some finite time for the velocity field to stabilize and for the pressure field to converge. Avoiding this phenomenon is generally not feasible for numerical wind synopsis simulations of unsteady wind flow. In order to avoid the influence of this phenomenon and to accurately evaluate the wind speed, the initial wind direction change over 360 degrees (400,000 time steps) is treated as the spin-up calculation. The computational results from the subsequent wind direction change of 360 degrees are used for the evaluation of the design wind speed discussed below.

3.3. Computational Results. Figure 5 shows the vertical profiles of wind velocity vectors (instantaneous values) at each of the turbine locations. These profiles were obtained after the wind direction was rotated over 360 degrees. This figure suggests that wind speed increases locally near the hubheight of each wind turbine due to topographical effects. Figure 6 shows that both the wind speed and turbulence intensity near the hubheight vary significantly according to the wind direction. In this figure, the wind speed at the hub height of a given turbine is illustrated in terms of the fractional increase of hubheight wind speed, that is, the ratio of the 10-minute-moving average value of the hubheight horizontal wind speed (scalar value) to the hubheight inflow horizontal wind speed which is free of topographical influence (the height correction coefficient $E_{p v}$ in Table 1),

$$
U_{\mathrm{EL}}=\sqrt{\bar{u}^{2}+\bar{v}^{2}}
$$

3.4. Evaluation of Design Wind Speed. In this subsection, the design wind speed is evaluated by calculating the fractional

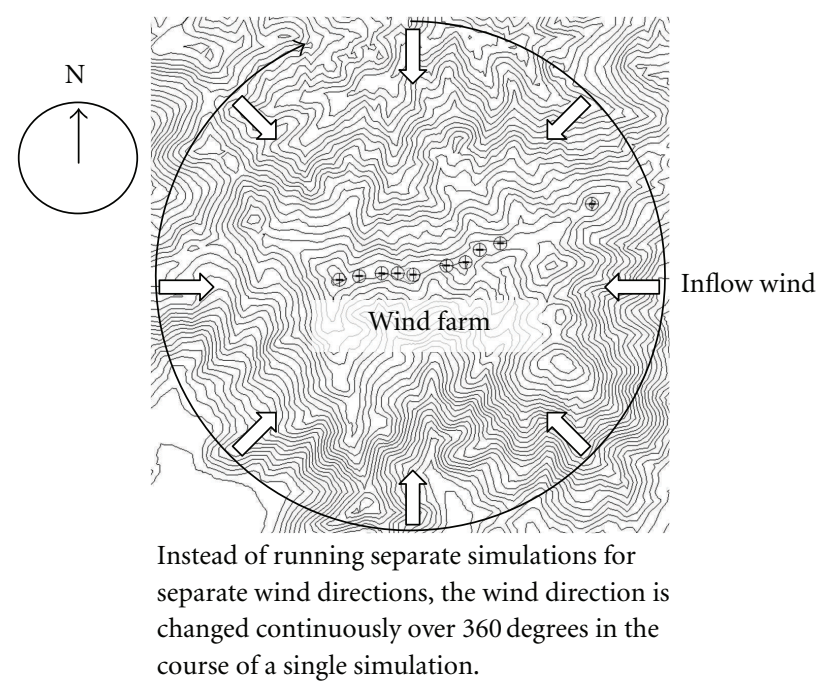

Figure 4: Continuous change of wind direction.

increase of hubheight wind speed from the simulation results. The design wind speed at the hubheight, denoted as $U_{h}$, can be estimated according to (3.1) in the literature [1] as:

$$
U_{h}=E_{t V} E_{p V} V_{0}
$$

where $E_{t V}$ is the fractional increase of hubheight wind speed, $E_{p V}$ is the height correction coefficient given in Figure 2 and Table 1 in Section 2.2, and $V_{0}$ is the design standard wind speed given in Article 87 of the Order for Enforcement of the Building Standards Act (for the target area of the current simulation, $V_{0}=34 \mathrm{~m} / \mathrm{s}$ ). Here, the maximum value of the fractional increase of hubheight wind speed in Figure 6(a) is used for $E_{t V}$, where $E_{t V}$ is defined as (the horizontal wind speed at the turbine hubheight)/(the wind speed at the hubheight) in Figure 2, Table 1. The value of $E_{t V}$ is 1.70 from Figure 6(a). For $E_{p V}$, the following value is used:

$$
E_{p V}=1.7(60 / 450)^{0.2}=1.14
$$

where the estimate is made for a $60 \mathrm{~m}$ hubheight using the relevant values for roughness classification III.

The design wind speed $U_{h}$ for the hubheight in the present study can be calculated as:

$$
U_{h}=E_{t V} E_{p V} V_{0}=1.70 \times 1.14 \times 34=65.89(\mathrm{~m} / \mathrm{s}) .
$$

However, the designer needs to take into account a safety factor in order to finalize the value of the design wind speed for the hubheight.

\section{Summary}

In the present study, the design wind speed of a wind turbine generator was evaluated for the hubheight. The evaluation was made using the fluid dynamics model RIAMCOMPACT, which allows analyses of unsteady turbulence fields. The wind speed and direction change subject to the 


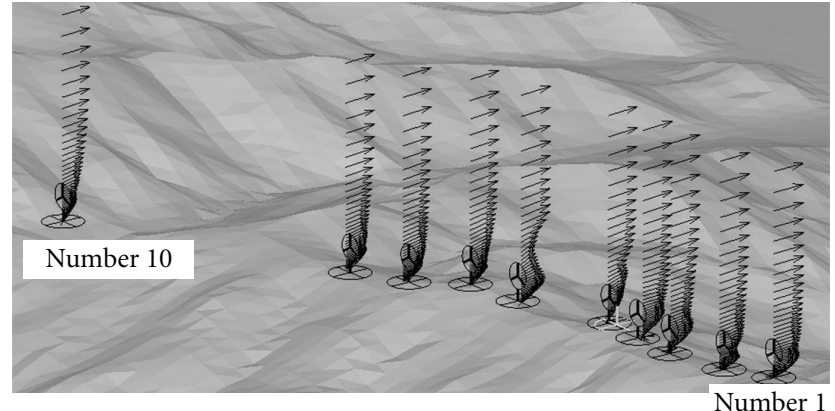

Figure 5: Vertical profile of wind velocity vectors at the location of each turbine. Northerly wind case. Instantaneous values.

influence of the surrounding topography is simulated precisely, and the simulation result is used for evaluating the design wind speed in the proposed technique as outlined below:

\section{Procedure for the Proposed Evaluation Technique:}

(1) Complex terrain information is constructed using data such as the 50-m-elevation data of the Geographical Survey Institute of Japan. Fine grid spacing is adopted near the turbines in order to reproduce the terrain features in detail. When complex terrain is included in the computational domain, a buffer zone with flat, almost zero elevation is set up around the computational domain, and the ground surface in the buffer zone is adjusted to connect smoothly to the complex terrain in the computational domain.

(2) Using previous literature [1], the vertical profile of the inflow wind speed is determined according to the surface roughness in the area surrounding the analysis domain.

(3) A simulation is performed during which the vertical profile of the wind speed determined in Step 2 is rotated continuously through 360 degrees.

(4) The fractional increase of hubheight wind speed is evaluated according to the literature [1], and the design wind speed is evaluated.

The proposed technique for the design wind speed evaluation was applied to a wind farm within Japan. A specific procedure for the design wind speed evaluation was laid out, and the design wind speed was evaluated for the turbine hubheight. The characteristics of the proposed technique include the following.

(i) Fine-scale wind speed and direction change that are subject to the influence of the surrounding topography on the wind field can be simulated more accurately than feasible with the technique in which simulations are performed for a specific number of wind directions, for example, 8 or 16 directions.

(ii) The proposed technique does not require the generation of new computational grids or spin-up calculations for wind field stabilization separately for each

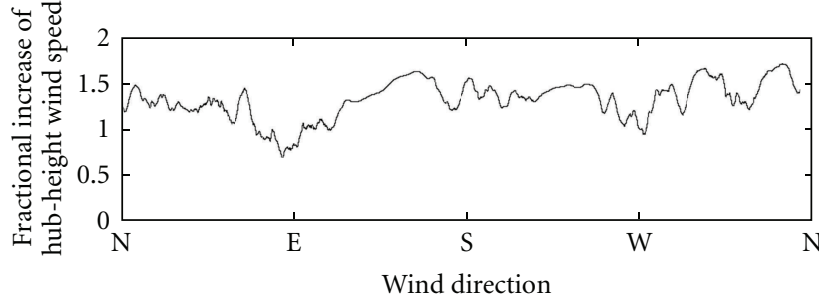

(a) Fractional increase of hubheight wind speed = (horizontal wind speed at turbine hubheight)/(horizontal wind speed at hubheight) from Figure 2 and Table 1

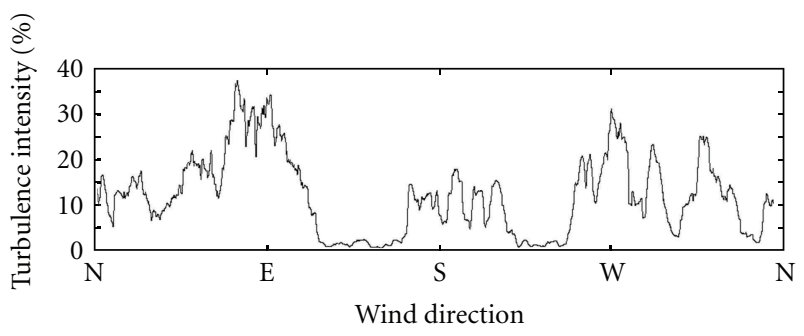

(b) Turbulence intensity (\%)

Figure 6: (a) 10-minute moving average values of the fractional increase of hubheight wind speed, and (b) corresponding turbulence intensity. Here, the results from Turbine no. 1 are given as an example.

of the predetermined wind directions. As a result, the simulation time can be reduced considerably.

Furthermore, possible applications of the present numerical wind synopsis prediction technique for unsteady wind flow which incorporates continuous wind direction change include:

(i) numerical site calibration (NSC) [9],

(ii) prediction of turbulence intensity over complex terrain,

(iii) coupling with the numerical results from a mesoscale meteorological model.

\section{Acknowledgments}

The present study was partially funded by (1) a Grantsin-Aid for Scientific Research for Young Researchers (A) for the fiscal year 2010 2011, "Development of a Local Wind Synopsis Prediction Technique for Improvement of the Atmospheric Environment in Mega-Cities in Asia and for the Effective Use of Wind Energy" (Principle Investigator: Takanori Uchida), (2) a 21st term research grant from the Research Foundation for Electrotechnology of Chubu: fiscal year 2010, "Development of a High-Accuracy Wind Turbine Micro-Siting Technique for the Dissemination of Wind Power Generation" (Principle Investigator: Takanori Uchida), and (3) a Research Grant from the Hatayama Cultural Foundation: fiscal year 2010, "Development of a Wind Turbine Micro-Siting Technique Contributing to the Appropriate Deployment of Wind Power Generation Facilities" (Principle Investigator: Takanori Uchida). Various 
datasets used in the present study were provided by Graham Li of Eurus Energy Japan Corporation. The authors wish to express our gratitude to the above-mentioned funding agencies and individuals.

\section{References}

[1] Guidelines for the Design of Wind Turbine Support Structures/ Commentary, Japan Society of Civil Engineers, 2007.

[2] Building Standard Law of Japan, 2000.

[3] Architectural Institute of Japan-Recommendations for Loads on Buildings / Commentary, 2004.

[4] T. Uchida, T. Maruyama, T. Takemi, Y. Oku, Y. Ohya, and Graham Li, "Proposal of designed wind speed evaluation technique in WTG installation point by using the meteorological model and CFD model," Journal of Wind Engineering and Industrial Aerodynamics, vol. 34, pp. 118-124, 2010 (Japanese).

[5] T. Uchida and Y. Ohya, "Micro-siting technique for wind turbine generators by using large-eddy simulation," Journal of Wind Engineering and Industrial Aerodynamics, vol. 96, no. 1011, pp. 2121-2138, 2008.

[6] T. Kajishima, "Finite-difference method for convective terms using non-uniform grid," Transactions of the Japan Society of Mechanical Engineers, Part B, vol. 65, no. 633, pp. 1607-1612, 1999.

[7] T. Kawamura, H. Takami, and K. Kuwahara, "Computation of high Reynolds number flow around a circular cylinder with surface roughness," Fluid Dynamics Research, vol. 1, no. 2, pp. 145-162, 1986.

[8] J. Smagorinsky, "General circulation experiments with the primitive equations, part 1, basic experiments," Monthly Weather Review, vol. 91, pp. 99-164, 1963.

[9] T. Sanada, M. Fujino, D. Matsushita et al., "Numerical site calibration on a complex terrain and its application for wind turbine performance measurements," in Proceedings of the European Wind Energy Conference (EWEC), pp. 156-160, 2006. 

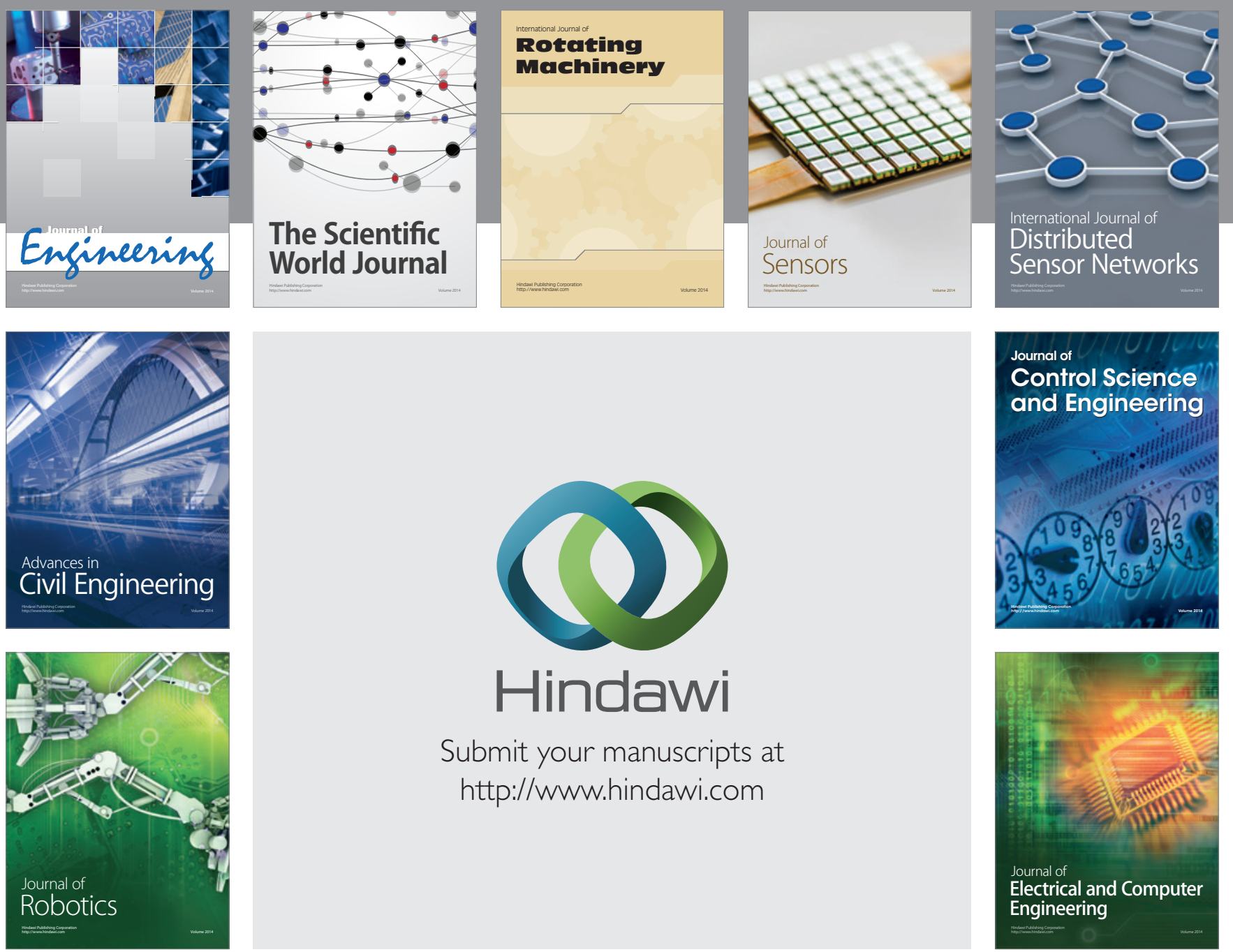

Submit your manuscripts at

http://www.hindawi.com
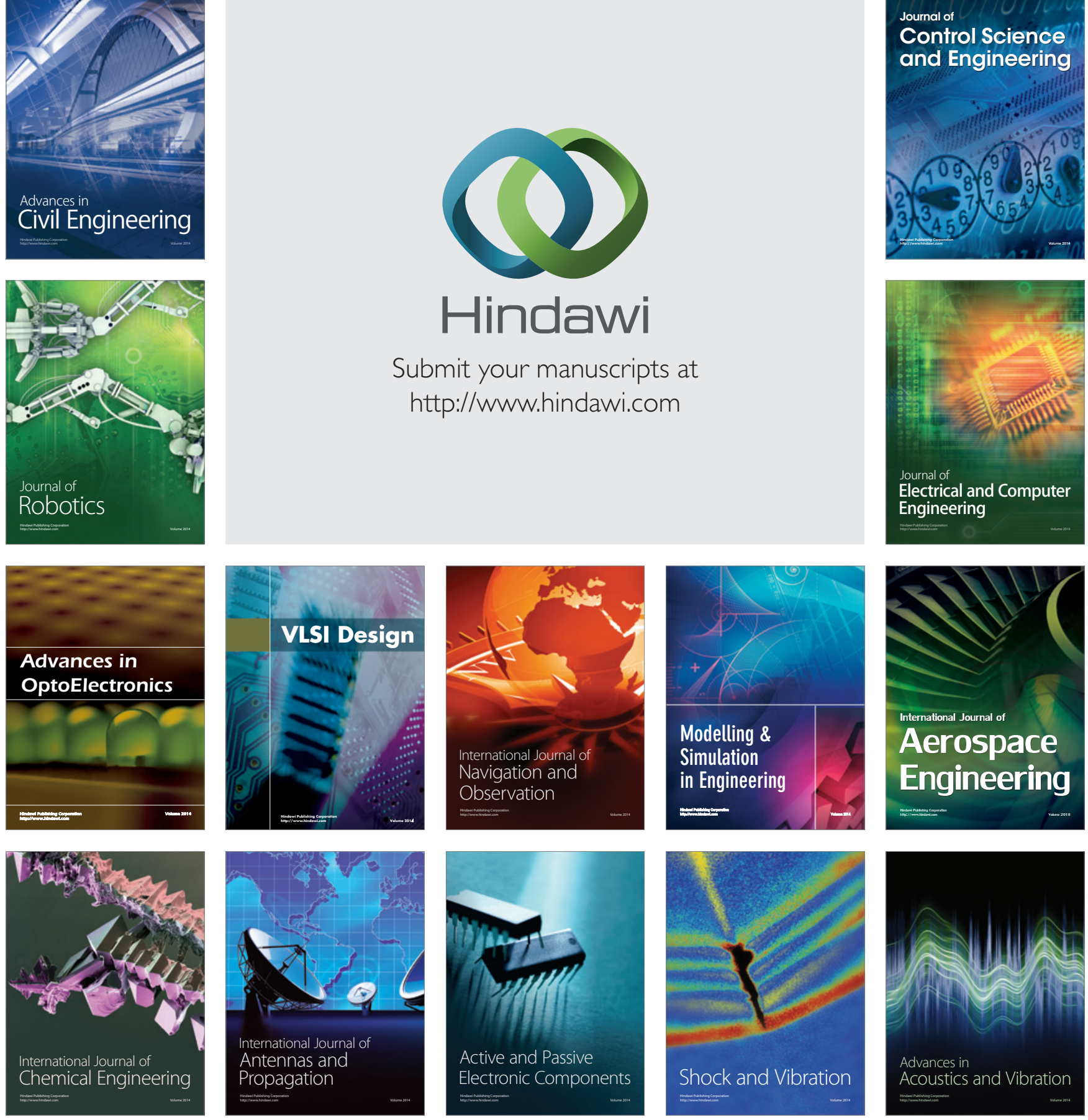